\title{
HIGH-RESOLUTION OBSERVATIONS OF THE M81 NUCLEUS
}

\author{
William C. Keel \\ Dept. of Physics and Astronomy \\ University of Alabama \\ Tuscaloosa, AL 35487-0324 \\ U.S.A.
}

The nucleus of the nearby Sb galaxy M81 has been shown to exhibit many of the features prominent in luminous active galactic nuclei: a variable, compact VLBI radio source (Kellermann et al. 1976, Jones, Sramek and Terzian 1981), a central variable X-ray source (Elvis and van Speybroeck 1982, Fabbiano 1988), and broad wings of Balmer emission similar in extent to those typical to Seyfert 1 nuclei (Peimbert and Torres-Peimbert 1981, Shuder and Osterbrock 1981). The nucleus also shows a narrow-line spectrum in many ways typical of LINERs (Heckman 1980), now known to be representative of early-type spiral nuclei in general.

The proximity of M81 offers a unique opportunity to study the environment of a (weak) active nucleus at unsurpassed linear resolution. This paper describes results of optical spectroscopy obtained in superb seeing, allowing a significant limit to be set on the mass of an unseen central object and revealing additional spectroscopic clues to the structure of the emission-line region. The limit on central mass then translates into an upper bound on the "duty cycle" of luminous active nuclei if objects like M81 are their relics seen during quiescent periods.

Stellar velocity dispersions near the nucleus were measured using an IPCS spectrum obtained with the 2.5-m Isaac Newton Telescope on La Palma, in seeing measured with the guide TV (and confirmed by a star in the slit) at 0.6 " FWHM. Each pixel subtends a spatial area of 0.7 by 1.0 arcsecond. The nucleus was nearly centered in a row of the spectrum; Fig. 1 shows the spectrum of this single row, along with an estimated starlight contribution and the derived emission spectrum. Velocity dispersions were derived in the Fourier domain, using the K star HD 85990 as a reference. Four rows around the nucleus have $\sigma_{v}=175 \pm 15 \mathrm{~km} / \mathrm{s}$, with the surrounding bulge just outside this having $\sigma_{v}=142 \pm 18$ $\mathrm{km} / \mathrm{s}$.

A straightforward analysis of the $\sigma_{v}$ profile has been done by resampling the bestfitting Dressler and Richstone (1988) model for M31 to simulate the distance of M81. This indicates that no unseen mass greater the $2 \times 10^{7} M_{\odot}$ is present, with a marginal and highly model-dependent value of $10^{7} M_{\odot}$ suggested. The implications are that, first, the mass of a central object is not directly related to the observed level of nuclear activity in this regime; that the efficiency of radiation during accretion must be greater than $10^{-5}$; and that, if M81 was ever a luminous quasar, it could not have been one for more than about $10^{8}$ years in all without violating this mass limit. If this object is typical, luminous quasar activity is highly episodic, in accord with the inference drawn from evidence for triggering by galaxy encounters. 


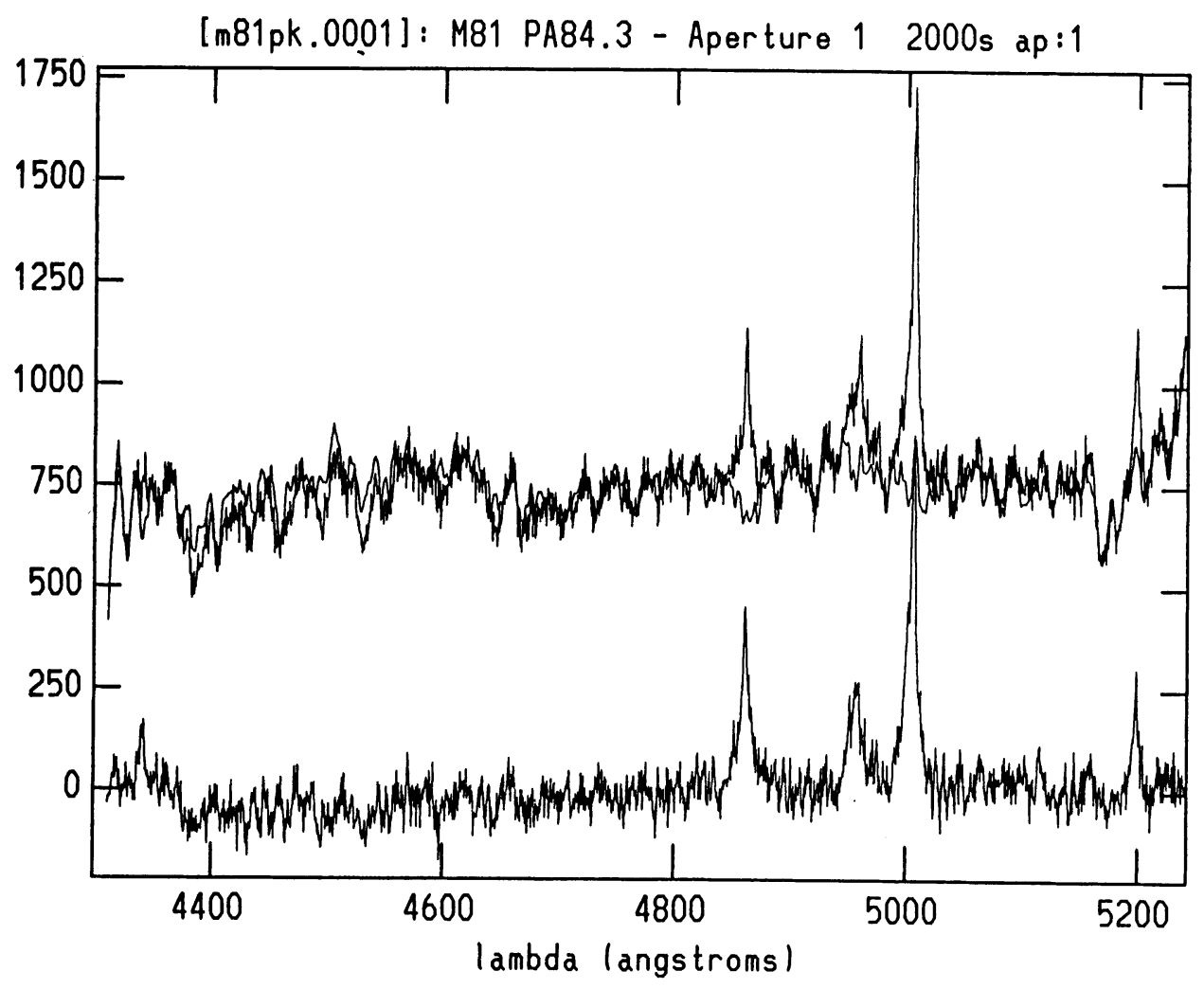

Fig. 1. IPCS spectrum of the inner $0.7 \times 1.0$ arcseconds of M81, along with an estimated starlight contribution and the derived emission-line spectrum.

The emission spectrum in the innermost region shows Fe VII $\lambda 5159$ emission, suggesting a region of intermediate density and linewidth. Evidence of a complex velocity and ionization structure on small scales comes from very narrow yet centrally concentrated [N I] $\lambda 5199$ emission and the apparent multicomponent nature of the [O III] and $\mathrm{H} \beta$ profiles.

I am grateful to M. Franx for providing the Fourier software, and to the EPSCoR program for support under grant RII-8610669. The Isaac Newton Telescope is operated by the Royal Greenwich Observatory on behalf of the UK SERC.

Dressler, A. and Richstone, D. 1988, Astrophys. J., 324, 701.

Elvis, M. and van Speybroeck, L. 1982, Astrophys. J. (Lett.), 257, L51.

Fabbiano, G. 1988, Astrophys. J., 325,544.

Heckman, T.M. 1980, Astron. Astrophys., 87, 152.

Jones, D.L., Sramek, R.A. and Terzian, Y. 1981, Astrophys. J., 246, 28.

Kellermann, K.I., Shaffer, D.B., Pauliny-Toth, I.I.K., Preuss, E. and Witzel, A. 1976, Astrophys. J. (Lett.), 210, L121.

Peimbert, M. and Torres-Peimbert, S. 1981, Astrophys. J., 245, 845.

Shuder, J.M. and Osterbrock, D.E. 1981, Astrophys. J., 250, 55. 